\title{
O CÁLCIO CONSUMIDO POR ADOLESCENTES DE ESCOLAS PÚBLICAS DE OSASCO, SÃO PAULO
}

\section{CONSUMPTION OF CALCIUM BY ADOLESCENTS FROM PUBLIC SCHOOLS EM OSASCO, SÃO PAULO, BRAZIL}

\author{
Barbara Regina LERNER' \\ Dóris Lúcia Martini LEI' \\ Sandra Pinheiro CHAVES' \\ Renata Damião FREIRE²
}

\begin{abstract}
RESUMO
No ano 2000 a população brasileira contará com mais de 14 milhões de idosos e a osteoporose se destaca como uma das enfermidades que afetará pelo menos 3 em cada 20 mulheres brasileiras. Uma das medidas preventivas é assegurar o consumo adequado de cálcio dietético para garantir que o indivíduo atinja o pico de massa óssea geneticamente determinado (que se dá entre os 25 e 30 anos), mantenha esta massa na idade adulta e apresente perda mínima na velhice. O objetivo do estudo foi o de conhecer e avaliar o consumo de cálcio por adolescentes de escolas públicas do Município de Osasco, tendo em vista a prevenção da osteoporose. Foi estudado o consumo alimentar de 323 alunos de $5^{a}$ e $8^{a}$ séries de 8 escolas localizadas nas regiões central e periférica do município. Utilizou-se a média de registro alimentar de 3 dias alternados. O consumo médio diário de cálcio e a porcentagem de cálcio oriunda de alimentos lácteos não foram significativamente diferentes entre homens e mulheres. Somente 6,2\% dos homens e 2,8\% das mulheres apresentaram consumo de cálcio acima de $1200 \mathrm{mg} /$ dia. Houve diferenças significativas entre as regiões geográficas com relação ao consumo de energia, de cálcio, assim como para a porcentagem de cálcio oriundos de alimentos lácteos e para a densidade do mineral na dieta. A média de cálcio ingerido do quartil mais alto (1015 mg/dia) não atinge a recomendação (1200 mg/dia). Os alimentos lácteos mais consumidos foram leite, queijo, sorvete e iogurte. O leite foi o alimento mais citado por todos os adolescentes, porém a quantidade difere entre os gêneros; as mulheres ingerem cerca de $190 \mathrm{ml}$ por dia enquanto os homens tomam $240 \mathrm{ml}$.O consumo de cálcio dos adolescentes está muito abaixo das recomendações atuais, refletindo-se na baixa densidade do mineral na dieta diária. Seria desejável uma densidade de pelo menos 550 mgCa/1000 Kcal para alcançar os 1200 mg diários recomendados. É necessário um esforço entre os profissionais da saúde no sentido de estimular o aumento do consumo de alimentos ricos em cálcio visando a prevenção da osteoporose e suas conseqüências.
\end{abstract}

Termos de indexação: cálcio, adolescente, osteoporose, prevenção \& controle.

\section{ABSTRACT}

In the year 2000 the Brazilian population will be about 14 million old citizens and osteoporosis will affect 3 in 20 Brazilian women. The prevention of osteoporosis consists of maximizing the peak of bone

1) Núcleo de Investigação em Nutrição, Instituto de Saúde da Secretaria de Estado da Saúde de São Paulo. Rua Santo Antonio, 590, 30 andar, 01314-000, São Paulo, SP, Brasil. Correspondência para/Correspondence to: B.R. LERNER.

2) Bolsista de Aperfeiçoamento do CNPq no Núcleo de Investigação em Nutrição, Instituto de Saúde da Secretaria de Estado da Saúde de São Paulo. 
mass genetically determined (that occurs from 25 to 30 years old), maintaining this achieved bone mass throughout adulthood and losing the minimum in old ages. The aim of this study was the evaluation of the calcium intake by adolescents from the city of Osasco (São Paulo, Brazil), looking forward to osteoporosis prevention. The food intake of 323 schoolchildren in the $5^{\text {th }}$ and $8^{\text {th }}$ grades from 8 schools located in the central and peripheral areas of the city was evaluated. The mean of the 3 alternate days dietary records was calculated. The mean daily intake of calcium and the calcium percentage from dairy foods were not significantly different for men and women. Only $6.2 \%$ of the men and $2.8 \%$ of the women consumed above $1200 \mathrm{mg} / \mathrm{d}$ of dietary calcium. There were significant differences in the energy and calcium intake and in the percentage of calcium from dairy foods and the mineral density of the diet in relation to the geographic areas. The mean calcium intake of the quarter of the population that showed the highest consumption (1 $015 \mathrm{mg} / \mathrm{d}$ ) did not meet the daily recommendation of $1200 \mathrm{mg}$. Milk was the dairy food that most of the adolescents referred to, but the quantities differed according to gender; women took $190 \mathrm{ml} /$ day and men $240 \mathrm{ml}$. The calcium intake of these adolescents was well below the present recommendations and so is the mineral density of their diets. It would be desirable an intake of, at least, $550 \mathrm{mgCa} / 1000 \mathrm{Kcal}$ to reach the daily recommendations. An effort should be made, by health professionals, to raise the intake of calcium-rich foods looking forward to the prevention of osteoporosis and its consequences.

Index terms: calcium, adolescent, osteoporosis, prevention \& control.

\section{INTRODUÇÃO}

O crescimento e desenvolvimento humano ocorrem por etapas. Os dois períodos de maior velocidade de crescimento se dão durante a vida intra-uterina até os dois anos de idade e da puberdade até a idade adulta dos 12 aos 20 anos (Hamill et al., 1979; Roche \& Himes, 1980). A adolescência é, portanto, profundamente marcada pelo intenso crescimento e desenvolvimento físico.

O esqueleto é o tecido de crescimento mais visível. Durante este período chega a triplicar de tamanho sendo necessário contar com um suprimento contínuo de cálcio e fósforo durante todo o seu desenvolvimento, pois cerca de $60 \%$ do peso do osso maduro é constituído por minerais, principalmente os dois citados (Peacock, 1991).

A única fonte de cálcio disponível para o organismo humano é aquele proveniente da dieta, sendo importante garantir uma ingestão mínima do mineral para o completo crescimento e maturação dos ossos. O pico de aquisição de massa óssea, geneticamente determinado, se dá até os vinte anos de idade quando 90\% do total é adquirido. Os outros $10 \%$ se completam até os 35 anos de idade.

Com o avançar da idade há uma diminuição progressiva da massa óssea que predispõe o indivíduo à osteoporose e suas conseqüências, estando associada a diversos fatores como raça, sexo, atividade física e à ingestão inadequada de cálcio por períodos prolongados.

O aumento da expectativa de vida das populações tem feito com que a osteoporose tenha uma distribuição universal, com altas estimativas de prevalência dado que é uma enfermidade relacionada ao envelhecimento. A expectativa de vida da população brasileira também está aumentando e apresenta uma variação de 63,4 anos em 1980 a 1985, até 72,1 esperada para os anos 2020 a 2025 (Kalache et al., 1987), incluindo-se, portanto, entre aquelas onde os males do envelhecimento estarão, com certeza, presentes.
Do ponto de vista econômico e social é uma enfermidade que traz grandes prejuízos, quer pela incapacitação dos pacientes quer pelo tratamento prolongado das fraturas decorrentes dela: um terço dos indivíduos que fraturam o colo do fêmur ficam incapacitados definitivamente para caminhar, enquanto que essas fraturas reduzem o tempo de vida de $10 \%$ a $20 \%$, ocorrendo a morte nos primeiros seis meses depois da fratura (Melton, 1988).

A osteoporose tem, portanto reconhecimento como enfermidade de importância social e econômica, que gera invalidez e custos elevados (Zerbini, 1987; Carneiro, 1988; Yabur, 1989; Kanis \& Pitt, 1992; World..., 1994; Fernandes et al., 1995).

Uma boa formação óssea tem sido vista, ultimamente, como um dos meios mais eficazes de prevenir a perda de massa óssea em idades mais avançadas (Matkovic, 1992), portanto, é importante adquirir o máximo da massa óssea possível durante o crescimento (Hui et al., 1990; Matkovic \& Ilich, 1993), uma vez que o período de maior retenção de mineral ocorre durante os anos da adolescência. Assim sendo, é necessário que haja um suprimento constante de cálcio dietético nesta fase do desenvolvimento humano para garantir a massa óssea máxima dentro do programa genético individual e a proteção desta massa acumulada em idades avançadas.

As recomendações de consumo diário de cálcio para a idade de 11 a 24 anos foram fixadas em $1200 \mathrm{mg} /$ dia pelo National Research Council em 1989 (National Research..., 1989), porém, a partir de uma reunião de consenso, realizada pelo National Institute of Health (National Institute...,1994), as recomendações para o consumo diário de cálcio para esse grupo etário, para ambos os sexos, passa a ser de 1200-1500 mg/dia.

O conhecimento do consumo diário de cálcio dos adolescentes brasileiros é, pois, de importância fundamental para o desenvolvimento de estudos epidemiológicos e para o estabelecimento de Programas 
Populacionais no sentido de manter uma adequação do consumo deste mineral com vistas à prevenção da osteoporose na população brasileira.

O objetivo deste estudo é o de conhecer e avaliar o consumo de cálcio de um grupo de adolescentes de escolas públicas do município de Osasco.

\section{CASUÍSTICA E MÉTODOS}

A amostra estudada é composta de alunos de quinta e oitava séries de oito escolas da Rede Oficial de Ensino do município de Osasco que foi pesquisada quanto à prevalência de anemia e consumo alimentar de ferro por Lerner (1994).

As escolas pertenciam à 2a Delegacia de Ensino e estavam localizadas em quatro Administrações Regionais de Ensino de Osasco (AR) segundo a situação de déficit estatural dos alunos ingressantes no primeiro ano do primeiro grau, conforme estudo realizado por Monteiro et al. (1989). Escolas das ARs 1 e 2 se localizavam em área de maior prevalência de déficit de crescimento do que as escolas da ARs 3 e 4.

Foram selecionadas duas escolas de cada AR, num total de oito escolas, correspondendo a $10 \%$ das escolas da rede pública de ensino de Osasco, na época. Destas escolas foram sorteadas uma classe de quinta e uma de oitava série, obtendo-se um total 509 alunos.

O consumo alimentar foi obtido através do registro dos alimentos ingeridos, por três dias alternados, incluindo eqüitativamente os sete dias da semana. Os alimentos foram registrados pelo estudante, em medidas caseiras, separados por refeição e local de consumo, em formulário próprio.

Ao definir o registro de três dias alternados, procurou-se detectar maior variabilidade de alimentos consumidos pela população, evitando-se o registro de "sobras" do dia anterior. Houve o cuidado de se incluir, nesses dias alternados, os sábados e os domingos que tiveram assim, a mesma oportunidade de serem registrados que os outros dias da semana.

Vários autores definem que três dias de levantamento é um número razoável para cobrir a quantidade e a variabilidade de alimentos consumidos por um grupo de indivíduos (Ferro-Luzzi, 1982; Persson \& Carlgren, 1984; Gunthrie \& Crocetti, 1985; Basiotis et al., 1987; Bingham, 1987).

Os dias da semana escolhidos para o registro foram selecionados da seguinte maneira: os formulários foram divididos em sete grupos (grupo 1: segunda-feira, quarta-feira e sexta-feira; grupo 2: terça-feira, quinta-feira e sábado; grupo 3: quarta-feira, sexta-feira e domingo; grupo 4: segunda-feira, quinta-feira e sábado; grupo 5: terça-feira, sexta-feira e domingo; grupo 6: segunda-feira, quarta-feira e sábado; grupo 7: terça-feira, quinta-feira e domingo), cada um destinado ao registro da alimentação relativa a três dias diferentes da semana, de tal maneira que a dieta de cada dia da semana foi, portanto, registrada por três grupos diferentes de jovens, em igual proporção aumentando assim, a possibilidade de detectar as variações do consumo alimentar decorrentes dos diferentes dias da semana, além daquelas inerentes à população (Schutz, 1982).

Os alimentos registrados foram transformados em gramas por meio da utilização dos pesos de medidas caseiras apresentados no programa de computação "Sistema de Apoio à Decisão em Nutrição", desenvolvido pelo Centro de Informática em Saúde da Escola Paulista de Medicina (EPM) e na "Tabela de Equivalência de Pesos em Grama dos Alimentos em Medidas Caseiras" do Departamento de Nutrição da Faculdade de Saúde Pública da Universidade de São Paulo.

Os cálculos para avaliar o teor de energia e de nutrientes foram feitos usando-se a tabela de composição de alimentos do Estudo Nacional da Despesa Familiar (ENDEF) (Fundação..., 1977) e do Instituto de Nutrición de Centro America y Panamá (INCAP) (Tabla..., 1966).

Foram considerados alimentos fonte de cálcio, para este estudo: queijo cremoso aromatizado com frutas (tipo danoninho), queijos, requeijão, doce de leite, mingau de aveia, pudim de pão e de leite, arroz-doce, iogurte, leite fermentado com lactobacilos, sorvete cremoso, leite em pó integral e desnatado e leite fluido tipos A, B, C e longa vida integral e desnatado.

O documento "Aplicações das Recomendações Nutricionais Adaptadas à População Brasileira" foi usado como referencial para a avaliação da adequação de ingestão de energia e dos outros nutrientes (Vannucchi et al., 1990).

Com relação à adequação de cálcio foi considerado o valor de 1200 mg/dia para homens e mulheres como o mínimo adequado.

Médias e desvios-padrão da quantidade de energia e dos nutrientes ingeridos durante os três dias de registro, por refeição e por dia, foram calculados para cada um dos indivíduos e para cada grupo de indivíduos, definidos por sexo e idade, e caracterização social. Diferenças de médias foram analisadas através do teste " $\mathrm{t}$ " para amostras independentes, com nível de significância $p<0,01$.

\section{RESULTADOS}

Dos 509 formulários entregues, foram devolvidos 380 , perfazendo $74,6 \%$ da amostra. As perdas foram maiores entre os homens $(44,0 \%)$ do que entre as mulheres $(31,0 \%)$ e nas idades mais jovens. Após análise de consistência foram usados 323 inquéritos de alunos que 
registraram os três dias de semana solicitados para este estudo, sendo 112 homens (34,7\%) e 211 mulheres (65,3\%) com idades médias de 13,7 \pm 1,5 anos e 14,0 \pm 1,6 anos, respectivamente.

O consumo energético médio foi de $2275 \pm$ $668 \mathrm{Kcal} /$ dia para os homens e $2009 \pm 566 \mathrm{Kcal} /$ dia para as mulheres, estando com $90 \%$ de adequação para ambos os gêneros.

O consumo médio diário de cálcio não foi significativamente diferente entre homens e mulheres, estando, nos dois casos, perto de 50,0\% daquele recomendado. Somente $6,2 \%$ dos homens e $2,8 \%$ das mulheres apresentaram consumo de 1200 mg/dia ou mais. A porcentagem de cálcio oriunda de alimentos lácteos foi de $56,1 \%$ para os homens e de $57,8 \%$ para as mulheres. Da mesma maneira, a densidade do mineral na dieta também não apresentou diferença entre os gêneros, embora mostre que as mulheres têm maior quantidade do mineral em 1000 kcal de sua alimentação (Tabela 1).

Tabela 1. Consumo diário de cálcio do grupo de adolescentes segundo gênero. Osasco, 1990.

\begin{tabular}{|c|c|c|}
\hline & Homens & Mulheres \\
\hline \multicolumn{3}{|c|}{ Consumo médio de cálcio } \\
\hline (mg/dia) & $628,85 \pm 353,82$ & $565,68 \pm 295,43$ \\
\hline \multicolumn{3}{|c|}{ Densidade de cálcio da } \\
\hline dieta (mg/1000kcal) & 278,52 & 286,97 \\
\hline
\end{tabular}

Há diferenças significativas entre as duas regiões tanto para o consumo de energia como para o consumo de cálcio. A região mais privilegiada mostra maior porcentagem de adolescentes que consomem cálcio igual ou acima da quantidade diária recomendada, assim como a porcentagem de cálcio oriundas dos alimentos lácteos e a densidade do mineral na dieta são significativamente maiores, ficando claro a desvantagem da região onde havia maior incidência de retardo de crescimento (AR1 e AR2) (Tabela 2).

Ao dividir a população estudada segundo os quatro quartis de consumo de cálcio, observamos na Tabela 3 as diferenças significativas entre o primeiro e o último quartil de consumo, tanto na média de consumo do mineral quanto na densidade do mineral da alimentação e na quantidade de energia proveniente dos alimentos lácteos.

Embora o último quartil de consumo englobe adolescentes que atingiram o consumo diário recomendado de cálcio, e por não serem eles a maioria, a média da quantidade de cálcio ingerida ainda está abaixo daquela recomendada, chegando a $84 \%$ de adequação. Observa-se, ainda, que os adolescentes do primeiro quartil apresentam um consumo muito pequeno de cálcio, ou seja, $25 \%$ da população estudada consome em média, somente um quinto do recomendado.

Ao verificar o consumo dos alimentos fonte de cálcio, dez adolescentes $(3,1 \%)$, sendo três homens e sete mulheres não referiram consumo de qualquer um desses

Tabela 2. Consumo alimentar diário dos adolescentes segundo regiões. Osasco, 1990.

\begin{tabular}{lcc}
\hline & $\mathrm{AR}_{1} \mathrm{e} \mathrm{AR}_{2}$ & $\mathrm{Ar}_{3}{\mathrm{e} \mathrm{AR}_{4}}$ \\
$\mathrm{n}=194$
\end{tabular}

${ }^{(*)}(\mathrm{p}<0,01)$

${ }^{(* *)}$ National Research... (1989), Vannucchi (1990)

${ }^{(* * *)}$ National Institute... (1994)

Tabela 3. Adolescentes segundo o primeiro e último quartis de consumo. Osasco, 1990.

\begin{tabular}{|c|c|c|}
\hline & 19 quartil & $4^{\circ}$ quartil \\
\hline Consumo energético médio (kcal) & $1742 \pm 551$ & $2439 \pm 621^{*}$ \\
\hline Consumo médio de cálcio (mg) & $242 \pm 81$ & $1015 \pm 256^{*}$ \\
\hline Densidade de cálcio da dieta (mg/1000 kcal) & 150,50 & $438,70^{*}$ \\
\hline$\%$ energia de alimentos lácteos & 5,67 & $21,90^{*}$ \\
\hline \% cálcio de alimentos lácteos & 42,20 & $68,70^{*}$ \\
\hline
\end{tabular}

${ }^{(*)}(\mathrm{p}<0,01)$ 
Tabela 4. Adolescentes segundo o consumo de alimentos lácteos. Osasco, 1990.

\begin{tabular}{|c|c|c|c|c|}
\hline $1^{\mathrm{o}}$ quartil & leite & sorvete & queijo & iogurte \\
\hline$\%$ de adolescentes & 53 & 42 & 28 & 0 \\
\hline Média de consumo/dia & $77,8 \mathrm{ml}$ & $41,5 \mathrm{~g}$ & $13,7 \mathrm{~g}$ & 0 \\
\hline Medida caseira & 1/3 de copo médio & $2 / 3$ de bola & 1 fatia pequena & 0 \\
\hline \multicolumn{5}{|l|}{$4^{\circ}$ quartil } \\
\hline$\%$ de adolescentes & 97 & 67 & 70 & 41 \\
\hline Média de consumo/dia & $332,5 \mathrm{ml}$ & $64,2 \mathrm{~g}$ & $29,7 \mathrm{~g}$ & $56,9 \mathrm{~g}$ \\
\hline Medida caseira & 11/2 copo médio & 1 bola & 2 fatias médias & $1 / 2$ copinho comercial \\
\hline \multicolumn{5}{|c|}{ Consumo acima de 1200 mgCa } \\
\hline$\%$ de adolescentes & 100,0 & $53,8 \%$ & $61,0 \%$ & 46,0 \\
\hline Média de consumo/dia & $481 \mathrm{ml}$ & $154 \mathrm{~g}$ & $48 \mathrm{~g}$ & $76 \mathrm{~g}$ \\
\hline Medida caseira & 2 1/2 copos médios & $21 / 2$ bolas & $31 / 2$ fatias médias & $2 / 3$ copinho comercial \\
\hline
\end{tabular}

alimentos. Para os outros 313 adolescentes, perto de $60 \%$ do cálcio ingerido foi oriundo de alimentos lácteos, embora, como já referido, a grande maioria tenha um consumo inadequado de cálcio. Destes adolescentes somente 13 alcançaram $1200 \mathrm{mg}$ de cálcio ao dia ou mais, sendo 7 homens e 6 mulheres.

Dos alimentos que foram considerados fontes, os mais consumidos foram leite, queijo, sorvete e iogurte. A Tabela 4 mostra a porcentagem de adolescentes que consumiu estes alimentos, a quantidade média de consumo obtida através dos registros alimentares e a sua correspondência em medida caseira, tanto para os 13 que consumiram uma quantidade igual ou maior do que a recomendada assim como para o primeiro e último quartil de consumo da população de adolescentes.

A quantidade média dos alimentos lácteos mais consumidos pelos adolescentes do quartil de consumo mais alto correspondem a 998 mg de cálcio, ou seja $98 \%$ do cálcio ingerido por esses adolescentes é proveniente destes quatro alimentos. Mesmo assim a média de consumo fica um pouco abaixo da recomendação de 1200 mg/dia do mineral.

O leite foi o alimento mais citado por todos os adolescentes; $83,4 \%$ das mulheres e 79,3 dos homens tomam leite, porém a quantidade difere entre os gêneros. As mulheres ingerem cerca de $190 \mathrm{ml}$ por dia enquanto os homens tomam $240 \mathrm{ml}$.

\section{DISCUSSÃO}

O consumo médio diário estimado de cálcio dos adolescentes estudados está muito abaixo das recomendações atuais (52,4\% de adequação), porém não diferem muito da situação encontrada entre outras populações de adolescentes.
Jaime et al. (1997), estudando jovens universitários paulistas de 18 a 27 anos encontraram um consumo médio diário de 754,08 mg/dia (62,84\% de adequação). Gambardella (1995), estudando a dieta de estudantes de 10 a 18 anos do período noturno de escolas do município de Santo André, verificou que mais de $60 \%$ das mulheres e entre 48 a $60 \%$ dos homens referiram ingerir menos de duas porções de alimentos lácteos diariamente, quantidade insuficiente para alcançar as recomendações do mineral. Barr (1994), ao estimar o consumo de cálcio de 785 alunos de escolas urbanas do Canadá encontrou os homens consumindo em média $1146 \mathrm{mg} /$ dia e as mulheres 815 $\mathrm{mg} /$ dia, sendo que $59 \%$ do cálcio foi obtido do leite; mais da metade dos indivíduos apresentaram consumo abaixo das recomendações.

Nos Estados Unidos da América, de 55,0 a 75,0 do cálcio dietético é proveniente de alimentos lácteos - leite, queijos e iogurtes (Allen, 1982; Block et al., 1985). Porcentagem semelhante foi encontrada entre os alunos deste estudo, onde esses três alimentos citados estão entre os quatro alimentos lácteos consumidos em maior número de vezes pelos adolescentes paulistas, sendo que os alimentos lácteos foram responsáveis por 56,5\% do cálcio dietético presente na alimentação.

Outros dados americanos referentes ao grupo de mulheres de 15 a 18 anos revelam um consumo de cálcio que variou de 602 mg/dia no ano de 1992 estando abaixo das recomendações de consumo para todas as idades; $98 \%$ da amostra se encontrava com o consumo abaixo dos $1200 \mathrm{mg} /$ dia recomendados (Albertson et al., 1997). Neste mesmo grupo, $44 \%$ do cálcio dietético foi oriundo de alimentos lácteos, sendo que o consumo médio de leite (alimento mais consumido) foi de $169 \mathrm{~g}$ de leite fluido ao dia, consumo este inferior ao consumo dos adolescentes de Osasco aqui estudados que foi de $190 \mathrm{ml}$ de leite ao dia, o que corresponde a um copo médio mal cheio de leite. 
O consumo de cálcio dos adolescentes de Osasco, à semelhança de outros estudos, deixa muito a desejar. Esse baixo consumo de alimentos ricos em cálcio se reflete na baixa densidade do mineral na dieta diária. Seria desejável uma densidade de pelo menos 550 mgCa/1000 Kcal para, com um consumo de $2200 \mathrm{Kcal}$, alcançar os 1200 mg diários recomendados.

Já é consenso entre os especialistas que indivíduos que não alcançam a maior densidade de massa óssea durante o pico de formação que se dá nos anos da adolescência encontram-se em maior risco de vir a desenvolver osteoporose em idades mais avançadas (Heaney, 1992; Schaafsma, 1992; Matkovic et al., 1993).

A atual recomendação para a ingestão de produtos lácteos que se encontram nos guias alimentares adotados no Brasil e em outros países é de 2 a 3 porções diárias. Um copo médio de leite (200 ml) contém 320 mg de cálcio; 15 gramas de queijo (uma fatia média), $480 \mathrm{mg} ; 120 \mathrm{ml}$ de iogurte (um copinho comercial) $568 \mathrm{mg}$ e uma bola de sorvete $90 \mathrm{mg}$. Para atingir os $1200 \mathrm{mg}$ recomendados seria necessário a ingestão de pelo menos três porções e dependendo dos alimentos até quatro porções de alimentos fonte de cálcio diariamente.

É necessário, portanto, que haja um esforço entre os profissionais da área da saúde no sentido de estimular o aumento do consumo de alimentos ricos em cálcio entre os adolescentes com o objetivo de prevenir esta doença degenerativa e suas conseqüências, uma vez que até hoje não foi confirmada a possibilidade de deposição de maiores quantidades de cálcio na idade adulta como uma correção da falta de cálcio dietético nas idades de formação do pico da massa óssea.

\section{AGRADECIMENTOS}

Às alunas do Curso de Nutrição das Faculdades Integradas São Camilo: Alessandra Prandini Romano, Daniela Sossai Rizo e Gabriela Halpern, pela contribuição na digitação e análise dos dados.

\section{REFERÊNCIAS BIBLIOGRÁFICAS}

ALBERTSON, A.M., TOBELMANN, R.C., MARQUAT, L. Estimated dietary calcium intake and food sources for adolescent female: 1980-1992. Journal of Adolescent Health, New YorK, v.20, n.1, p.20-26, 1997.

ALLEN, L.H. Calcium bioavailability and absorption: a review. American Journal of Clinical Nutrition, Bethesda, v.35, p.783-808, 1982.

BARR, S.I. Associations of social and demographic variables with calcium intakes of high school students. Journal of the American Dietetic Association, Chicago, v.94, n.3, p.260-269, 1994.
BASIOTIS, P.P., WELSH, S.O., CRONIN, F.J., KELSAY, J.L., MERTZ, $W$. Number of day of food intake records required to estimate individual and group nutrient intakes with defined confidence. Journal of Nutrition, Bethesda, v.117, p.1638-1641, 1987.

BINGHAM, S.A. The dietary assessment of individuals: method, accuracy, new techniques and recommendations. Nutrition Abstracts and Reviews, Aberdeen, v.57, n.10, p.705-742, 1987.

BLOCK, G., DRESSER, C.M., HARTMAN, A.M., CARROL, M.D. Nutrient sources in the American diet: quantitative data from the NHANES II survey. I. Vitamins and minerals. American Journal of Epidemiology, Baltimore, v.122, n.1, p.13-26, 1985.

CARNEIRO, R.A. Repercussões socioeconômicas da osteoporose no Brasil: estimativa de custos. Arquivos Brasileiros de Medicina, Rio de Janeiro, v.62, n.1, p.65-69, 1988.

FERNANDES, C.E., RIBEIRO, R.M., MELO, N.R., PINOTTI, M. Osteoporose. Revista Brasileira de Medicina, São Paulo, v.52, p.1170-1186, 1995.

FERRO-LUZZI, A. Meaning and constrain of energy intake studies in free-living populations. In: HARRISON, G.A. (ed). Energy and effort. London : Taylor \& Francis, 1982. p.115-135.

GAMBARDELLA, A.M.D. Adolescentes, estudantes de período noturno: como se alimentam e gastam suas energias. São Paulo, 1995. 81p. Tese (Doutorado) - Faculdade de Saúde Pública, Universidade de São Paulo, 1995.

GUNTHRIE, H., CROCETTI, A. Variability of nutrient intake over a 3-day period. Journal of the American Dietetic Association, Chicago, v.85, n.3, p.325-327, 1985.

HAMILL, P.V.V., DRIZD,T.A., JOHNSON, C.L., REED, R.B., ROCHE, A.F., MOORE, W.M. Physical growth: National Center for Health Statistics - percentiles. American Journal of Clinical Nutrition, Bethesda, v.32, p.607-629, 1979.

HEANEY, R.P. Calcium in the prevention and treatment of osteoporosis. Journal of Internal Medicine, Oxford, v.231, n.1, p.169-180, 1992.

HUI, S.L. SLEMENDA, C.W., JOHNSTONJR., C.C. The contribution of bone loss to postmenopausal osteoporosis. Osteoporosis International, London, v.1, n.1, p.30-34, 1990.

INSTITUTO BRASILEIRO DE GEOGRAFIA E ESTATÍSTICA (IBGE). Tabelas de composição dos alimentos. Rio de Janeiro, 1977. 201p. (Estudo Nacional da Despesa Familiar, v.3; publicações especiais, t.1).

JAIME, P.C., MOTOIE, E., CERVATO, A.M. Relação cálcio/fósforo na dieta habitual de adultos jovens. In: CONGRESO DE LA SOCIEDAD LATINOAMERICANA DE NUTRICIÓN "DR. ABRAHAM HORWITZ", 1., 1997, Guatemala. Anais... Guatemala, 1997. p.150.

KALACHE, A., VERAS, R.P., RAMOS,L.R. O envelhecimento da população mundial, um desafio novo. Revista de Saúde Pública, São Paulo, v.21, n.3, p.200-10, 1987.

KANIS, J.A., PITT, F.A. Epidemiology of osteoporosis. Bone, New York, v.13, p.7S-15S, 1992. Supplement.

LERNER, B.R. A alimentação e a anemia carencial em adolescentes. São Paulo, 1994. 90p. Tese (Doutorado) - Faculdade de Saúde Pública, Universidade de São Paulo, 1994.

MATKOVIC, V. Calcium intake and peak bone mass. New England Journal of Medicine, Boston, v.327, n.2, p.119-120, 1992. 
MATKOVIC V., ILICH, J.Z. Calcium requirements for growth: are current recommendations adequate? Nutrition Reviews, New York, v.51, n.6, p.171-180, 1993.

MELTON III. L.J.Epidemiology of fractures. In: RIGGS, B.L, MELTON III, L.J. (eds). Osteoporosis: etiology, diagnosis and management. New York : Raven Press, 1988. p.133-154.

MONTEIRO, C.A., LEI, D.L.M., MONDINI, L., CORDELINI, S., BARATHO, R.M., CHAVES, S.P., BONALDO, E. Coleta e análise da altura de escolares em um sistema de vigilância nutricional: desenvolvimento de metodologia, implantação e avaliação. São Paulo: USP, 1989.55p. (Relatório Técnico; Mimeografado).

NATIONAL INSTITUTE OF HEALTH. Optimal calcium intake. Journal of the American Medical Association, Chicago, v.272, n.24, p.1942-1948, 1994.

NATIONAL RESEARCH COUNCIL (USA). Recommended Dietary Allowances. 10.ed. Washington DC : National Academy Press, 1989. 284p.

PEACOCK, M. Calcium absorption efficiency and calcium requirements in children and adolescents. American Journal of Clinical Nutrition, Bethesda, v.54, p.261S-265S, 1991. Supplement.

PERSSON, L.A., CARLGREN, G. Measuring children's diets: evaluation of dietary assessment techniques in infancy and childhood. International Journal of Epidemiology, London, v.13, p.506-517, 1984.

ROCHE, A.F., HIMES, J.H. Incremental growth charts. American Journal of Clinical Nutrition, Bethesda, v.33, n.3, p.2041-2052, 1980.
SCHAAFSMA, G. The scientific basis of recommended dietary allowances for calcium. Journal of Internal Medicine, Oxford, v.231, n.1, p.187-194, 1992.

SCHUTZ, H.G. Prediction of nutritional status from food consumption and consumer attitude data. American Journal of Clinical Nutrition, Bethesda, v.35, p.1310-1318, 1982.

TABLA de composición de alimentos para uso en America Latina, INCAP - ICNND. In: BURTON, B.T. Nutrición humana: un tratado completo sobre nutrición en la salud y la enfermedad. Washington DC : OPS, 1966. p.461-600. (OPS. Publicación Cientifica, 146).

VANNUCCHI, H., MENEZES, E.W., CAMPANA, A.O., LAJOLO, F.M. Aplicações das recomendações nutricionais adaptadas à população brasileira. Cadernos de Nutrição, São Paulo, v.2, n.1, p.1-156, 1990.

WORLD HEALTH ORGANIZATION. Assessment of fracture risk and its application to screening for postmenopausal osteoporosis. Geneva, 1994. (Technical Report Series, 843)

YABUR, J.A. Epidemiologia, importancia social y costo de la osteoporosis. Archivos Venezolanos de Farmacologia y Terapéutica, Caracas, v.8, n.2, p.130-135, 1989.

ZERBINI, C.A.F. Por que o interesse na osteoporose? Revista Paulista de Medicina, São Paulo, v.105, p.229-300, 1987.

Recebido para publicação em 16 de outubro de 1998 e aceito em 4 de maio de 1999. 